\title{
Kahoot! no Ensino Superior: razões para a gamificação das aulas por meio de uma ferramenta digital de quizzes
}

\author{
Kahoot! in Higher Education: \\ reasons for the gamification of classes through \\ a digital quizzes tool
}

\author{
Aline Espig \\ Universidade Regional de Blumenau \\ Maria José Carvalho de Souza Domingues \\ Universidade Regional de Blumenau
}

Resumo: Áreas tradicionais de ensino são percebidas como monótonas e pouco práticas. A utilização da gamificação tende a tornar o ensino ativo e reduzir o distanciamento entre alunos e professores. $\mathrm{O}$ objetivo deste estudo foi responder quais são as características percebidas pelos professores usuários do Kahoot! que contribuem para a sua adoção como apoio ao ensino em administração e contabilidade. A teoria utilizada nesta análise foi a da Difusão Tecnológica, que aponta a existência de características e atributos que afetam sua adoção. A pesquisa foi aplicada a professores do estado de Santa Catarina - Brasil. O retorno foi de 30 professores usuários do Kahoot!. Os dados foram analisados através de regressão linear múltipla. Os resultados apresentam que entre "vantagem relativa", "compatibilidade", "experimentação", "facilidade de uso", "imagem", "uso voluntário", "visibilidade" e "demonstração de resultado", somente, a característica facilidade de uso foi considerada fator de influência para a adoção ao uso do Kahoot!.

Palavras-chave: Kahoot!. Difusão tecnológica. Gamificação.

\begin{abstract}
Traditional teaching areas are perceived as monotonous and impractical. The use of gamification tends to make teaching active and reduce the gap between students and teachers. The objective of this study is to analyze which characteristics are perceived by lecturers who use Kahoot! that contribute to its adoption as a support tool for teaching in administration and accounting. The theory used in this analysis was the Technological Diffusion theory, which points out that there are characteristics and attributes that affect its adoption. The research was applied to lecturers in the state of Santa Catarina - Brazil. There were thirty respondent lecturers who use Kahoot!. The data were analyzed applying multiple linear regression. The results show that among "relative advantage", "compatibility", "experimentation", "ease of use", "image", "voluntary use", "visibility", and "demonstration of results", only the "ease of use" characteristic was considered a factor of influence for the adoption of the use of Kahoot!.
\end{abstract}

Keywords: Kahoot!. Technological diffusion. Gamification. ESPIG, Aline; DOMINGUES, Maria José Carvalho de Souza. Kahoot! no Ensino Superior: razões para a gamificação das aulas
por meio de uma ferramenta digital de quizzes. Informática na Educação: teoria \& prática, Porto Alegre, v. 23, n. 2, p. 123141 , maio./ago. 2020 . 


\section{Introdução}

As tecnologias de informação e comunicação estão possibilitando novas formas de aprendizagem (ARETIO, 2019). Essas novas formas vêm apresentando-se como algo indispensável, tendo em vista a dificuldade que os métodos de ensino convencionais têm em atrair a atenção dos estudantes (NASU; AFONSO, 2018).

Neste cenário, o chamado "ensino convencional" não é mais suficiente para atrair a atenção dos alunos, sendo este composto por diversos fatores como a infraestrutura ultrapassada das salas de aula (CUNHA et al., 2013), a discrepância existente entre os métodos de ensino e as práticas do mercado de trabalho e, principalmente, o distanciamento entre os estudantes e os professores (BORNAL et al., 2018). Por esses motivos, buscar práticas docentes que considerem os estilos de aprendizagem de cada aluno é fundamental, indo ao encontro dos chamados métodos ativos de aprendizagem, ou seja, os métodos que visam tirar a passividade dos estudantes (BORNAL et al., 2018).

O uso das Tecnologias da Informação e Comunicação (TICs) como metodologias ativas pode ser feito através de gamificação, com a ferramenta de quizzes, utilizando o sistema de resposta do estudante (SRE). Nos quizzes, aplicam-se perguntas e respostas utilizando os dispositivos móveis dos estudantes, nos quais o docente apresenta uma questão (geralmente de múltipla escolha), e os estudantes respondem por meio do aplicativo. Assim, o software recebe a resposta pela internet, dando um feedback aos alunos e professores (NASU; AFONSO, 2018; ALMENARA; ROBLES, 2018). Enquanto em um método de ensino convencional o feedback pode vir apenas na avaliação formal, com o uso de quizzes, o docente pode ter um panorama da aprendizagem dos estudantes ao seu método de ensino no mesmo dia.

Além disso, Bornal et al. (2018) apontam que a interação do formato gamificado de aplicativos de quizzes apresenta um efeito de engajamento nos estudantes, relaxamento e até de prazer para algumas pessoas, principalmente, as que têm uma rotina cansativa. Esses benefícios são advindos da recompensa recebida (pontuação), característica marcante de todo jogo. Assim, as inovações tecnológicas na educação e a aplicação de quizzes com utilização de smartphones ou laptops pelos estudantes vêm apresentando efeitos positivos (DABBAGH; FAKE; ZHANG, 2019). Um dos recursos mais utilizados atualmente - e que usa o sistema SRE em formato gamificado - é o ambiente de jogo chamado "Kahoot!". Em 2019, mais de 2,5 bilhões de pessoas, de mais de 200 países, já haviam jogado Kahoot! (VICK, 2019).

O Kahoot! é uma ferramenta de jogo gratuita para gamificação e consiste em uma plataforma on-line para a realização de testes de múltipla escolha (GUARDIA, 2019). Wang e Tahir (2020) apontam que, na utilização do Kahoot!, a sala de aula é transformada temporariamente em um game show, o professor é o apresentador e os alunos são os candidatos, sendo esse cenário aprimorado a partir de meios de áudio e gráficos, pontos, placares e pódio, que tendem a contribuir para uma experiência de aprendizagem divertida e positiva. 
Conquanto o aplicativo seja bastante utilizado, em uma revisão da literatura de Wang e Tahir (2020) sobre o Kahoot!, percebeu-se que ainda há espaço para muitos estudos empíricos, entre eles, destaca-se a percepção de alunos e professores.

Quanto aos professores, a adoção ou não de tecnologias como o Kahoot! está associada aos motivos ou às características pelos quais tomam ou não a decisão de utilizá-las, tal adoção ou aceitação permeia a teoria da difusão da inovação tecnológica (TDI) de Rogers (1983). Diversas pesquisas (DAVIS et al.,1989; MOORE; BENBASAT, 1991; IGBARIA et al., 1995; AGARWAL; PRASAD， 2000; VENKATESH; DAVIS, 2000; LEWIS; AGARWAL; SAMBAMURTHY, 2003; VENKATESH et al., 2003; COMPEAU; MEISTER; HIGGINS, 2007; PÉREZ et al., 2012) abordam a adoção e a inovação tecnológica em relação a diversos tipos de inovação, tendo os pesquisadores o intuito de compreender as características relacionadas ao processo de aceitação de tecnologia na prática ou intenção de uso no ambiente de trabalho e/ou em tarefas produtivas (LEAL, 2012). Porém os resultados de pesquisas empíricas não são homogêneos.

Dessa forma, embora a gamificação aparente ser promissora na educação, há a necessidade de mais evidências empíricas de sua utilidade e capacidade de aumentar o interesse de quem a utiliza em um contexto educacional (SILVA; RODRIGUES; LEAL, 2019). Destaca-se, ainda, a importância desses estudos no contexto brasileiro, em que se verifica a falta de um ambiente que propicie o engajamento do aluno (TALOMEI, 2017). Para que os professores de fato venham a utilizar a ferramenta de gamificação (que pode ser uma opção para o aumento do interesse dos alunos), é necessário entender quais características os influenciam. Não foram encontradas pesquisas que investigassem essas características na utilização do Kahoot! em nenhum contexto.

Aponta-se, também, que, nos cursos de administração e contabilidade, suas disciplinas carecem de tecnologia e conteúdo prático na visão dos alunos, o que faz com que estes fiquem desinteressados, por considerarem o processo de ensino monótono (NASU; NOGUEIRA, 2019). Nessas disciplinas, a gamificação é um grande desafio para a educação, particularmente, em instituições de Ensino Superior (IES), em um contexto tão tradicional, como é o caso de ambos os cursos mencionados, além de outras disciplinas (LOPES et al., 2019). Nestas disciplinas, já foram apontadas evidências de que em cursos de contabilidade, a aplicação de estratégias de gamificação acabam por aumentar o nível de entendimento, participação individual e em grupo e interação (GÓMEZ; MONROY, 2018).

De acordo com Cunha et al. (2013), para os alunos de contabilidade, os atributos considerados prioritários no aprimoramento do curso de ciências contábeis são a utilização de laboratório para resolução de exercícios, modernidade e a infraestrutura das salas de aula. Nesta disciplina, o uso de novas tecnologias propicia que os discentes interajam com demais alunos (DE VARGAS; SCHERER; GARCIA, 2020). Percebe-se que a gamificação, aliada a tecnologia, além de ser um desafio no ensino de administração e contabilidade, por conta dos temas e métodos tradicionais de ensino, é também uma tendência percebida e buscada pelos alunos.

Então, este artigo tem a seguinte questão problema: Quais são as características percebidas pelos professores usuários do Kahoot! que contribuem para a sua adoção como ferramenta de apoio ao ensino em administração e contabilidade? Assim, o objetivo desta pesquisa foi o de 
identificar as principais características percebidas no uso do Kahoot!, nesse sentido, levando em consideração os aspectos que contribuem para a sua utilização como ferramenta de apoio aos professores do Ensino Superior de administração e contabilidade.

Similarmente à proposta do presente artigo, Pérez et al. (2012) investigaram a adoção da inovação tecnológica "Moodle" por professores universitários, assim, analisando a percepção das características levantadas por Rogers (1983) e Moore e Benbasat (1991), e constataram que facilidade de uso, experimentação, vantagem relativa e uso voluntário são as que levam os professores a utilizarem a plataforma.

Gunter e Braga (2018) ainda apresentam, em sua pesquisa na área das ciências humanas, que professores relatam que a falta de equipamento escolar e a necessidade de um currículo de tecnologias emergentes podem indicar um baixo grau de compatibilidade, o que apontaria que um novo sistema de valores é necessário para sua adoção.

Outra pesquisa que analisou a questão da adoção da inovação tecnológica por professores de administração e contabilidade foi realizada por Leal (2012), que constatou que as características percebidas pelos professores como influenciadoras da adoção e do uso da inovação tecnológica de ambiente virtual de aprendizagem (AVA) são, respectivamente, a compatibilidade, vantagem relativa e a demonstração de resultado. Já a facilidade de uso, experimentação, visibilidade, imagem, uso voluntário e tempo de experiência não apresentaram significância.

Percebe-se, assim, que, quanto à percepção das características da TDI que influenciam a adoção a uma inovação tecnológica no ensino, os resultados divergem conforme a inovação, e não se percebe uma concordância entre os autores em pesquisas empíricas. Ainda quanto à adoção de inovações tecnológicas na educação com base nas características apontadas por Rogers (1983), Gunter e Braga (2018) revelam que, apesar da adoção de dispositivos móveis pela sociedade, ainda precisamos saber se os professores percebem esses artefatos como uma inovação e qual é o seu potencial para o desenvolvimento e a prática do professor, o que pode estar influenciando os resultados de pesquisas empíricas.

Sendo assim, além do preenchimento de lacunas, esta pesquisa visa servir como fonte de informação para formuladores de aplicativos, dirigentes de instituições de ensino, professores e investigadores com o intuito de intensificar características que favorecem ao uso do aplicativo de jogo Kahoot!, e, portanto, aumentar o engajamento dos alunos e propiciar respostas rápidas de desempenho destes.

\section{Referencial Teórico}

Frente ao desafio atual da educação, que traz consigo elevados índices de evasão e repetência (VALENTE, 2014), tem-se um cenário no qual a tecnologia vem ganhando espaço. Para que seja possível enfrentar as dificuldades em manter o engajamento dos alunos neste cenário, são necessários novos métodos de aprendizagem (TOLOMEI, 2017).

Dabbagh, Fake e Zhang (2019) realizaram uma pesquisa em universidades dos Estados Unidos (EUA) e constataram que os alunos consideram de extrema importância o uso de 
dispositivos móveis para apoiar estudos, tais como laptops e smartphones, além da utilização de bases para pesquisa com o fim de auxiliar no desenvolvimento.

Desse modo, a cada dia, os professores precisam reinventar-se para manter os alunos engajados, e o modelo tradicional de ensino, que considera que todos têm o mesmo potencial e a mesma forma de aprender, perde espaço para novas metodologias que exploram a colaboração, a exploração, a investigação e a "mão na massa", ou seja, as metodologias ativas (OLIVEIRA NETO; GOMES; TITTON, 2017). Essas metodologias têm o intuito de propiciar ao aluno, além da absorção e retenção de informação, um papel ativo para, assim, compreenderem, elaborarem novos conhecimentos e, ainda, aplicá-los a situações concretas (VALENTE, 2014). Nesse sentido, tencionar a transformação é imperioso para ajudar o estudante a entender que ele é o principal responsável por sua formação (ANTUNES; NASCIMENTO; QUEIROZ, 2019).

Nos últimos dez anos, as tecnologias foram inseridas nesse ambiente de tal maneira que, atualmente, $87 \%$ das universidades utilizam as TICs para suporte de ensino (ALMENARA; ROBLES, 2018). Ou seja, se até há pouco tempo a utilização da tecnologia era considerada diferenciada, agora, faz parte de todo o contexto acadêmico. Conforme apontado por Aretio (2019), importantes organizações (como ONU e Unesco) destacam a necessidade de integrar TIC em todos os níveis educacionais para que, desse modo, os alunos possam beneficiar-se dessas ferramentas para o desenvolvimento da vida, do emprego, da governança, do lazer e da saúde.

Nesse contexto, os professores precisam saber que os métodos tradicionalmente utilizados nem sempre são os que mais atraem os alunos, logo, por conta disso, é necessário chamar atenção dos alunos mediante novas formas de didática (SILVA; RODRIGUES; LEAL, 2019), com isso, podendo reduzir a forte "crise motivacional na educação" (TOLOMEI, 2017). Uma das ferramentas que vêm chamando atenção nas novas metodologias de ensino aliadas à tecnologia é a "gamificação".

\subsection{Gamificação na educação e o Kahoot!}

A gamificação utiliza a competitividade como diferencial para estimular a motivação na educação. Além de ajudar a cooperar e comunicar (SILVA; RODRIGUES; LEAL, 2019), é considerada estratégia para o engajamento em sala de aula (TOLOMEI, 2017).

A gamificação se popularizou em 2010, em sala de aula, e é aplicada na promoção da educação utilizando elementos e design de jogos em um contexto que não necessariamente é um jogo (SILVA; RODRIGUES; LEAL, 2019). Embora a utilização dessas ferramentas em sala de tenham se popularizado há pouco tempo, já se cita que tal metodologia tende, também, a promover a participação, o envolvimento e a redução do medo de errar dos alunos (SILVA; RODRIGUES; LEAL, 2019).

As gamificação na educação apresenta premiações e recompensas para reconhecer a melhoria no desempenho do aluno de acordo com uma classificação que avalia as habilidades e os níveis de conhecimento apresentados pelos jogadores (SILVA; RODRIGUES; LEAL, 2019). Apontam-se quatro pontos principais na aplicação da gamificação (TALOMEI, 2017): 
- Tarefas realizadas são convertidas em pontos;

- O progresso dos alunos é relacionado aos níveis do jogo;

- Os feedbacks são necessários durante o jogo;

- Os erros são uma forma de testar outras formas de resolver os mesmos problemas. 
Exemplos empíricos do uso da gamificação são evidenciados por Talomei (2017) ao apresentar casos de aplicação nos EUA e no Brasil. Ambas as aplicações apresentaram resultados positivos, pois destacam o aumento na participação, na motivação e nas notas nos EUA. Já no Brasil, destaca-se também como resultado, além da melhora na motivação, o aumento da leitura, que traz consigo consequências positivas em interpretação (TALOMEI, 2017).

Um dos aplicativos mais utilizados na educação é o Kahoot!. Nos últimos anos, esta plataforma tornou-se uma ferramenta de gamificação digital para resposta dos alunos, e é usada para envolvê-los a partir de questionários (DELLOS, 2015). O Kahoot! consiste em uma plataforma on-line de aprendizado baseada em jogos, usada para revisar ou avaliar o conhecimento dos alunos de uma forma diferente dos meios comuns, sendo percebida como uma "pausa" das atividades tradicionais (WANG; TAHIR, 2020). A plataforma é gratuita e propicia um ambiente para testes de múltipla escolha (GUARDIA, 2019). A ferramenta pode ser encontrada no formato da $w^{*}{ }^{*}$ e no aplicativo móvel, para Android ou IOS.

Conforme apresentado por Wang e Tahir (2020), o Kahoot! foi o primeiro SRE projetado para fornecer uma experiência de jogo usando os princípios de design de jogos da teoria da motivação intrínseca de Malone (1981), e fluxo de jogo de Sweetser e Wyeth (2005). Sendo assim, o Kahoot! é uma combinação do uso de respostas do público e dramatizações usando recursos de vídeo e audiovisuais (WANG; TAHIR, 2020). O conceito de Kahoot! é combinar um SRE, a infraestrutura técnica existente nas instituições de ensino, o fato de os alunos estarem usando seus próprios dispositivos digitais, redes sociais e jogos em uma plataforma de aprendizado audiovisual (WANG; TAHIR, 2020).

Desde que a plataforma foi lançada, em 2013, estudos se concentram no efeito do Kahoot! na sala de aula; e, entre os resultados encontrados na maioria das pesquisas, tem-se que o aplicativo pode apresentar um efeito positivo no desempenho de aprendizagem, na dinâmica em sala de aula, nas atitudes e na ansiedade dos alunos (WANG; TAHIR, 2020).

\subsection{Difusão da Inovação}

A teoria da difusão da inovação (TDI) entrou em voga em meados dos anos de 1930 (ROGERS, 1983). Essa "difusão da inovação" refere-se ao processo de sua comunicação em determinado contexto social, envolvendo indivíduos e grupos, geralmente, integrantes de uma organização (ROGERS, 2003). Nesse contexto, a adoção de uma inovação também é um processo, em que os indivíduos e grupos decidem ou não pelo seu uso, de acordo com sua visão de utilidade, podendo, assim, ser aceito ou rejeitado.

Dos principais autores que investigaram a respeito das características percebidas pelos usuários na adoção de uma inovação tecnológica, de acordo com Pérez et al. (2012), destacamse Rogers (1983) e Moore e Benbasat (1991). Estes desenvolveram um instrumento para avaliação das percepções que um indivíduo pode ter sobre as características de uso de uma inovação: 
- Vantagem relativa: considera o nível em que uma inovação é entendida como melhor do que seu precursor (ROGERS, 1983).

- Compatibilidade: considera o nível em que uma inovação é entendida como consistente com valores, necessidade e experiências dos adotantes potenciais (ROGERS, 1983).

- Experimentação: considera o nível em que uma inovação pode ser experimentada antes de sua adoção (ROGERS, 1983).

- Facilidade de uso: considera o nível em que uma inovação é entendida como fácil de usar (MOORE; BENBASAT, 1991).

- Imagem: considera o nível em que a utilização de uma inovação é entendida como fator para melhorar a/o imagem/status do indivíduo em um sistema social (MOORE; BENBASAT, 1991).

- Uso Voluntário: considera o nível de percepção de uso espontâneo de uma inovação (MOORE; BENBASAT, 1991).

- Visibilidade: considera o nível em que uma inovação se torna aparente para outros indivíduos dentro de uma organização (MOORE; BENBASAT, 1991).

- Demonstração de resultado: considera o nível em que os resultados do uso de uma inovação são percebidos como tangíveis (MOORE; BENBASAT, 1991).

De acordo com as características apresentadas, nessa teoria, tem-se que as características inerentes à própria inovação, assim como os elementos ligados aos fatores organizacionais internos, são as que podem aumentar a taxa de usuários adotantes de uma inovação tecnológica ou não, ao longo do tempo (ROGERS, 1983). Então, o aumento da taxa de adoção é o ponto central da TDI (ROGERS, 1983).

\section{Metodologia}

O presente estudo buscou identificar as principais características percebidas para a difusão do ambiente de jogo Kahoot! no ensino de administração e contabilidade, para tanto, levando em consideração os aspectos que contribuem para sua utilização como ferramenta de apoio aos professores do Ensino Superior. Este estudo se caracteriza como de tipo quantitativo, de caráter descritivo.

Os dados foram coletados por meio de um questionário on-line. O questionário aplicado, que foi adaptado de Pérez et al. (2012), considera 4 questões para a caracterização da amostra e 40 questões referentes à adoção a inovação, as quais contemplam as características percebidas pelos indivíduos: vantagem relativa, compatibilidade, experimentação (ROGERS, 1983), facilidade de uso, imagem, uso voluntário, visibilidade e demonstração de resultado (MOORE; BENBASAT, 1991).

Quanto à aplicação dos questionários, destaca-se que foram enviados questionários a todos os coordenadores de curso de administração e contabilidade de instituições de Ensino Superior vinculadas ao sistema ACAFE - Associação Catarinense das Fundações Educacionais, para que replicassem a pesquisa ao quadro de professores das instituições. O número total de instituições 
contatadas foi 108. O endereço ao qual os e-mails foram enviados foram obtidos através de consulta aos websites das instituições. Solicitou-se, aos coordenadores, que o questionário fosse enviado e respondido por professores que utilizam o aplicativo Kahoot!. Foram respondidos e retornados 30 questionários válidos, que, posteriormente, foram submetidos à validação por meio de regressão linear múltipla.

Destaca-se que a amostra que respondeu ao questionário era diversificada entre professores de administração e contabilidade, sendo que $46,7 \%$ dos respondentes eram professores de administração, $26,7 \%$ eram professores de contabilidade e $26,7 \%$ lecionavam nos dois cursos professores de administração e contabilidade.

Questionou-se a idade dos respondentes e, entre os professores usuários do Kahoot! respondentes, 3,3\% apresentavam idade entre 18 e 25 anos; 13,3\%, de 26 a 30 anos; 20\%, acima de 50 anos; $26,7 \%$, entre 31 e 40 anos; e a maior parte, entre 41 e 50 anos, assinalando $36,7 \%$ dos respondentes.

Já quanto ao nível de formação dos respondentes, a grande maioria $(66,7 \%)$ dos respondentes tinha mestrado, seguida de doutorado $(23,3 \%)$ e especialização (10\%). Nenhum dos respondentes desta pesquisa lecionava apenas com título de graduação.

Para a análise de dados, inicialmente, foi realizada uma análise de confiabilidade das questões por meio do teste de Alfa de Cronbach. Posteriormente, realizou-se um agrupamento das questões de cada característica a partir da técnica de Análise de Componentes Principais (ACP). No intuito de identificar a existência de alguma influência dessas características na adoção ao uso do Kahoot!, os dados foram importados para o software IBM SPSS $®$ versão 22 e submetidos a uma equação de regressão linear múltipla.

Considerou-se a média das dimensões utilizadas no questionário on-line para medir as características da adoção à inovação como variáveis independentes, e a adoção ao uso do Kahoot!, propriamente dito, como variável dependente na equação de regressão, que compreende um conjunto de técnicas estatísticas utilizadas para modelar relações entre variáveis, predizendo o valor das variáveis dependentes (ou de resposta) a partir de um conjunto de variáveis independentes, também chamadas de preditoras (MAROCO; GARCIA-MARQUES, 2006). Assim, o resumo do modelo é apresentado na figura 1. 
Figura 1 - Modelo de pesquisa

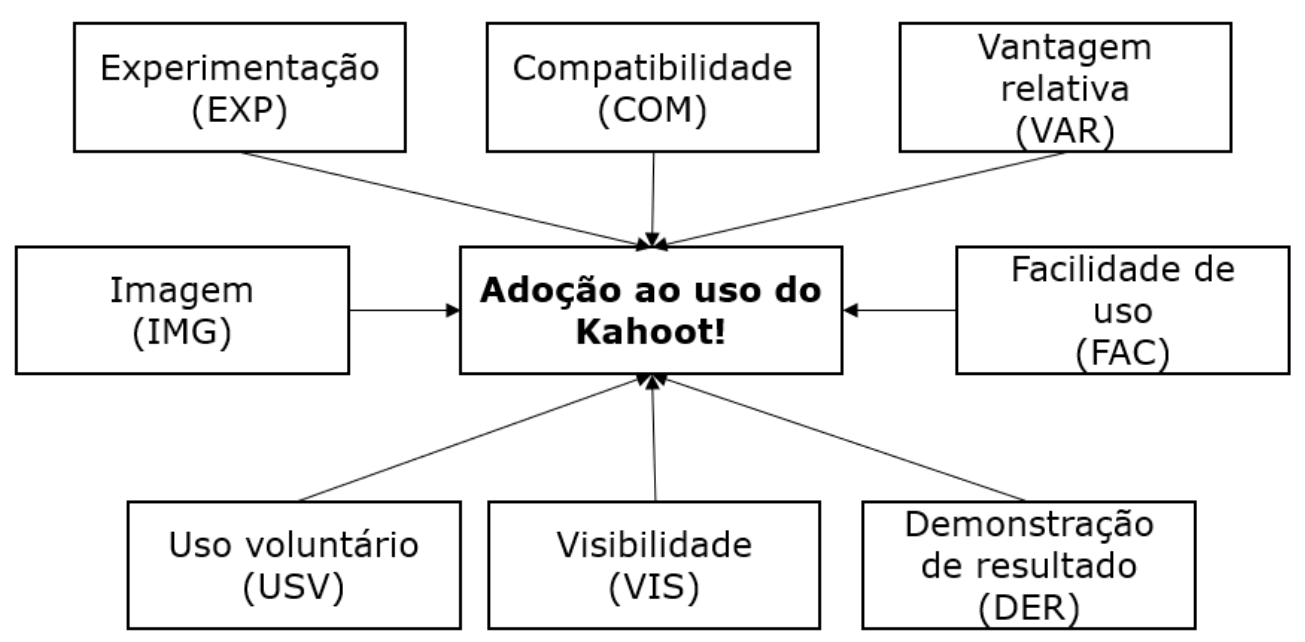

Fonte: autores (2020)

Com a análise da regressão linear múltipla, verificou-se o impacto das variáveis independentes na variável dependente. Na sequência, apresentam-se os resultados da aplicação da equação de regressão, bem como a discussão de seus resultados.

\section{Análise dos resultados e discussão}

\subsection{Análise de resultados}

Visando responder à pergunta de pesquisa foram realizadas análises de regressão linear múltipla. Para tanto, em um primeiro momento, foram aplicados os testes de confiabilidade das questões investigadas por meio do teste de Alfa de Cronbach (a). Este indicador refere-se à "fiabilidade" dos indicadores, ou seja, a capacidade de esta ser consistente (MAROCO; GARCIAMARQUES, 2006). As referências para este indicador variam de acordo com diferentes autores. Para amostragens de 25-50 indivíduos, Davis (1964) aponta que o valor aceitável deve ser > 0,5. Já Nunally (1978) apresenta que, em investigações preliminares, o valor deve ser $>0,7$. Sendo assim, nesta pesquisa, o valor do $a>0,7$ foi definido como referência. Então, foram analisadas todas as questões de cada uma das características investigadas, excluindo-se, portanto, questões que faziam com que a característica considerada tivesse $a<0,7$.

Tabela 1 - Alfa de Cronbach

\begin{tabular}{lcccc}
\hline Característica & Questões totais & a (Inicial) & Questões removidas & a (Final) \\
\hline Adoção ao uso & 3 & 0,667 & 1 & 0,932 \\
Vantagem relativa & 5 & 0,882 & 0 & 0,882 \\
Compatibilidade & 3 & 0,860 & 0 & 0,860 \\
Experimentação & 3 & 0,715 & 0 & 0,715
\end{tabular}




\begin{tabular}{lllll}
\hline Facilidade de uso & 4 & 0,977 & 0 & 0,977 \\
Imagem & 3 & 0,731 & 0 & 0,731 \\
Uso voluntário & 2 & 0,462 & 0 & 0,462 \\
Visibilidade & 3 & 0,280 & 1 & 0,785 \\
Dem. de resultado & 4 & 0,912 & 0 & 0,912 \\
\hline
\end{tabular}

Fonte: autores (2020)

No tocante ao padrão adotado ( $a>0,7$ ), duas questões foram removidas para que a confiabilidade fosse alcançada (uma questão em relação à "adoção ao uso"; e uma questão em relação à "visibilidade"). Porém, nas questões que compunham a característica "uso voluntário", mesmo que com uma das duas questões removidas, o valor do a não foi atingido com o mínimo, pois, conforme a tabela 1 , atingiu somente 0,462 .

Como consequências da falta de confiabilidade desta característica individualmente, teve-se uma probabilidade elevada de ela não se apresentar como válida ou, ainda, o erro nos procedimentos de regressão ser elevado, assim, fazendo com que não fosse significativa (MAROCO; GARCIA-MARQUES, 2006). Portanto, esta variável manteve-se no modelo e teve sua significância avaliada nas etapas de análise ulteriores, sendo excluída quando não alcançou significância.

Após esse procedimento, agrupou-se as questões que compunham cada uma das características. Também, foram gerados escores fatoriais para cada dimensão pelo método de análise de componentes principais (ACP), que consiste em juntar variáveis correlacionadas, formando combinações lineares independentes (MAROCO, 2018).

Em sequência, aplicou-se o teste ANOVA (tabela 2). O intuito do teste foi confirmar a possibilidade de utilizar a regressão linear múltipla, ou seja, testar o efeito das variáveis independentes ("vantagem relativa", "compatibilidade", "experimentação", "facilidade de uso", "imagem", "uso voluntário", "visibilidade" e "demonstração de resultado") sobre a variável dependente (adoção ao uso). Para que fosse possível validar a aplicação da regressão linear múltipla, o $p$-valor ou significância da ANOVA deveria ser < 0,05 (MAROCO, 2018). Considerando que o resultado da ANOVA foi de 0,001, entende-se que se deu uma relação estatisticamente significativa (neste caso, existindo regressão).

Tabela 2 - Regressão Linear Múltipla parte 1

\begin{tabular}{ccccc}
\hline $\begin{array}{c}\text { Sig. } \\
\text { ANOVA }\end{array}$ & $\mathbf{R}$ & R quadrado & R quadrado ajustado & Durbin Watson \\
\hline $0,001^{\mathrm{b}^{*}}$ & $0,817^{\mathrm{a}}$ & 0,668 & $0,541^{* *}$ & 2,315 \\
\hline
\end{tabular}

$(*)$ Sig. Anova $<0,05=$ significante $(* *) \mathrm{R}^{2}>0,50=$ Ajustamento do modelo adequado Fonte: Os autores (2020).

Na continuidade da análise, foi realizado o teste de Durbin Watson. A partir desse teste, objetivou-se avaliar se existia autocorrelação serial entre os resíduos ou erros (FIELD, 2009). Para concluir que não existia autocorrelação, o valor de referência deveria ser próximo a 2 
(FÁVERO et al., 2009). O resultado desse teste se mostrou favorável (2,315, conforme tabela 2$)$, então, não se verificou autocorrelação serial entre os resíduos; e o modelo, por sua vez, revelouse adequado.

Após a constatação da possibilidade de concretização da regressão por meio do teste ANOVA e Durbin Watson, foram avaliados os resultados da regressão. Maroco (2018) enfatiza a análise do coeficiente de determinação $\left(R^{2}\right)$. Esse coeficiente é utilizado como uma das medidas para avaliar a qualidade de ajustamento do modelo.

Conforme a tabela 3, esta pesquisa teve como resultado de $R^{2}$ ajustado o valor de 0,541 . Os valores de referência para este indicador dependem da subjetividade do estudo, porém, de acordo com Maroco (2018), o ideal em ciências sociais é que seja no mínimo 0,5. Sendo assim, percebe-se que o modelo possui qualidade adequada para a análise dos resultados da regressão.

Ao considerar o coeficiente de determinação $R^{2}$, infere-se que as variáveis independentes ("vantagem relativa", "compatibilidade", "experimentação", "facilidade de uso", "imagem", "uso voluntário", "visibilidade" e "demonstração de resultado") influenciaram até $54,1 \%$ da variável dependente adoção ao uso do Kahoot!. Embora se tenha essa conclusão, é necessário avaliar cada uma das variáveis independentes separadamente para verificar qual sua relação com a variável dependente. Para que uma variável seja considerada significativa, o $p$-valor ou significância deve ser < 0,05 (HAIR JR. et al., 2005). Nesse sentido, a partir da análise da significância das variáveis apresentados na tabela 3 , percebe-se que a variável facilidade de uso teve um ótimo ajustamento do modelo (sig. 0,002), e a variável compatibilidade apresentou significância (sig. 0,049). Porém as demais características analisadas no mesmo modelo não obtiveram significância.

Tabela 3 - Regressão Linear Múltipla parte 2

\begin{tabular}{lcccc}
\hline Variáveis & B não padronizado & B padronizado & Sig & VIF \\
\hline VAR & $-0,351$ & $-0,053$ & 0,697 & 1,131 \\
COM & 0,049 & 0,049 & 0,049 & 5,760 \\
EXP & $-0,132$ & $-0,132$ & 0,469 & 2,007 \\
FAC & 0,802 & 0,802 & $0,002 *$ & 3,287 \\
IMG & $-0,127$ & $-0,127$ & 0,409 & 1,444 \\
USV & $-0,053$ & 0,249 & 0,142 & 1,688 \\
VIS & 0,249 & 0,249 & 0,142 & 1,688 \\
DER & 0,372 & 0,372 & 0,469 & 2,007 \\
\hline
\end{tabular}

(*) Sig. $<0,05=$ significante

Fonte: autores (2020)

Realizou-se, ainda, a análise da influência da variância (VIF). A análise deste indicador tem a finalidade de medir o efeito das variáveis independentes sobre o coeficiente de regressão. Maroco (2018) e Fávero et al. (2009) apontam que o valor da VIF maior ou igual a 5 permite a conclusão de que há multicolinearidade entre as variáveis independentes do modelo proposto. Nesta 
pesquisa, a variável "compatibilidade" apresentou VIF de 5,760, então, revelando multicolinearidade. Sendo assim, a variável foi removida e a regressão foi realizada novamente.

Já na nova análise, apresentada na tabela 4, constatou-se que a significância da ANOVA era ideal $(0,000) ; \circ R^{2}$, adequado ( $R$ quadrado ajustado $=0,561$ ) e o Durbin Watson se encontrava próximo a $2(2,329)$ e de acordo com os valores de referência.

Tabela 4 - Regressão Linear Múltipla modelo 2 parte 1

\begin{tabular}{ccccc}
$\begin{array}{c}\text { Sig. } \\
\text { ANOVA }\end{array}$ & R & R quadrado & R quadrado ajustado & Durbin Watson \\
\hline $0,000^{\mathrm{b}}$ & $0,817^{\mathrm{a}}$ & 0,667 &, $561^{* *}$ & 2,329 \\
\hline
\end{tabular}

$(*)$ Sig. Anova $<0,05=$ significante $(* *) \mathrm{R}^{2}>0,50=$ Ajustamento do modelo adequado

Fonte: autores (2020)

Novamente, foram analisadas as variáveis isoladamente (Tabela 5). Percebeu-se, então, que, da mesma forma, a variável "facilidade de uso" foi a única que apresentou significância (sig. 0,001 , que é < 0,05). Em relação à multicolinearidade, com a exclusão da variável "compatibilidade", o modelo não apresentou mais problemas, ou seja, nenhuma das variáveis apresentou-se igual ou superior a 5 .

Tabela 5 - Regressão Linear Múltipla modelo 2 parte 2

\begin{tabular}{ccccc}
\hline Variáveis & B não padronizado & B padronizado & Sig & VIF \\
\hline VAR & $-0,356$ & $-0,356$ & 0,140 & 1,997 \\
EXP & $-0,134$ & $-0,134$ & 0,449 & 3,091 \\
FAC & 0,813 & 0,813 & $0,001 *$ & 1,438 \\
IMG & $-0,129$ & $-0,129$ & 0,391 & 1,113 \\
USV & $-0,056$ & $-0,056$ & 0,669 & 1,671 \\
VIS & 0,253 & 0,253 & 0,126 & 3,315 \\
DER & 0,411 & 0,411 & 0,080 & \\
\hline
\end{tabular}

(*) Sig. $<0,05=$ significante

Fonte: autores (2020)

A última interpretação dos dados da regressão analisados foi a análise dos coeficientes de regressão ( $B$ padronizado e $B$ não padronizado, apresentados na tabela 5). Esta análise permite iniciar uma discussão em relação aos resultados de cada uma das variáveis. De acordo com a única característica que de fato obteve significância (facilidade de uso), que considera o grau a partir do qual uma inovação é considerada fácil de ser usada (MOORE; BENBASAT, 1991), percebeu-se que essa característica revelou B padronizado e não padronizado positivo, ou seja, na percepção dos professores, quanto mais facilidade de uso do Kahoot!, maior é sua adoção.

\subsection{Discussão}


Ao considerar que, conforme a percepção dos professores, a facilidade de uso do Kahoot! é o que influencia sua adoção, percebe-se que esta evidência corresponde aos achados de Pérez et al. (2012) em relação à adoção da inovação tecnológica Moodle por professores. Embora a pesquisa desses autores tenha outra concepção de aplicação, obteve-se resultado similar nesta característica, também, em relação a uma inovação tecnológica. Os autores perceberam que, entre outras variáveis menos influentes, a facilidade de uso apresentou maior coeficiente de regressão em relação à adoção ao uso. Tal resultado corrobora as afirmações de Pérez (2006) e Rogers (2003), que justificam que quanto maior a facilidade de uso percebida na inovação, maior será a taxa de adoção (PÉREZ et al., 2012).

Justifica-se, ainda, essa influência da facilidade, pois, em países emergentes como o Brasil, uma das maiores dificuldades apresentadas pelos professores é a falta de disponibilidade de equipamentos e recursos tecnológicos (CUNHA et al., 2013). Porém essa barreira da falta de infraestrutura técnica existente nas instituições de ensino, com a utilização do Kahoot!, tende a ser quebrada pelo fato de os alunos estarem trazendo seus próprios dispositivos digitais (WANG; TAHIR, 2020).

Sobre as variáveis que não obtiveram significância, inicia-se com a variável "vantagem relativa", sendo que esta, conforme já mencionado, refere-se ao grau com o qual uma inovação é percebida como melhor do que sua precursora (ROGERS, 1983). Uma das possibilidades para essa baixa significância é o fato de que o Kahoot! foi o precursor como SRE, que forneceu uma experiência de jogo usando os princípios de design de jogos da teoria da motivação intrínseca, sendo o primeiro a utilizar o fluxo de jogo (WANG; TAHIR, 2020). Assim, o Kahoot! não pôde ser comparado com seu precursor, pois não existia outro aplicativo similar antes de sua criação.

Outra variável que não apresentou significância foi a compatibilidade. Essa característica se refere ao grau com o qual uma inovação é percebida como consistente com valores, necessidade e experiências dos adotantes potenciais (ROGERS, 1983), ou seja, neste estudo, é pertinente à percepção dos professores de que seus valores estão relacionados ao uso do Kahoot!, e estes, por esse motivo, adotam ou não a inovação tecnológica. Gunter e Braga (2018), em uma pesquisa sobre a adoção da inovação de dispositivos móveis por professores em ciências humanas, não obtiveram resultados positivos e apresentam que professores relataram que a falta de equipamento escolar e a necessidade de um currículo de tecnologias emergentes podem indicar um baixo grau de compatibilidade, o que apontaria que um novo sistema de valores é necessário para sua adoção.

Se essa verificação de compatibilidade foi percebida empiricamente em 2018, ela pode ser uma possível justificativa para a baixa significância no presente estudo. Tem-se que parte dos professores concordou que a compatibilidade influencia na adoção ao uso do Kahoot!; outros, não, e outra parte da amostra posicionou-se como indiferente. Nesta mesma linha, Hoerup (2001) descreve que cada inovação influencia as opiniões, as crenças, os valores e os pontos de vista dos professores sobre o ensino. Ou seja, Gunter e Braga (2018) apontam que, para aumentar a percepção da característica compatibilidade, seria necessário um novo sistema de valores, e possivelmente, parte da amostra da presente pesquisa já possui essa mentalidade. 
$\mathrm{Na}$ avaliação da adoção ao Moodle, Pérez et al. (2012) obtiveram significância na variável compatibilidade, porém a influência foi extremamente baixa na adoção ao uso. Os autores justificam esse resultado apresentando que boa parte dos professores que adotou o Moodle não teve a oportunidade de acessar outro tipo de ferramenta que pudesse suprir suas necessidades, sendo ainda considerado um sistema pouco explorado em funções mais avançadas.

Caberia, então, às instituições implantarem um sistema de novos valores, conforme sugerido por Gunter e Braga (2018), influenciando os professores, já que o Kahoot! é apresentado como uma forma de alternar as atividades de ensino tradicionais, assim, fazendo com que alunos fiquem mais engajados, e os professores possam avaliar o processo de aprendizagem (WANG; TAHIR, 2020).

A variável experimentação, que também não foi confirmada como característica que influencie na adoção ao uso da inovação tecnológica, consiste no grau com o qual uma inovação pode ser experimentada antes de sua adoção (ROGERS, 1983).

Kebritchi (2010) aponta que, em uma pesquisa realizada com professores de matemática, na qual se verificou as características da adoção de jogos educacionais em computadores, os professores sugeriram, em entrevista, que, quanto à experimentação, seria fator para a adoção de jogos educacionais a disponibilização de uma versão de teste por um período limitado de tempo, apontando que isso seria apropriado para definirem se, de fato, adotariam a inovação. Porém esse cenário pode não favorecer aos usuários do Kahoot! pelo fato de ser uma ferramenta gratuita, e os testes serem totalmente liberados. Então, por conta do Kahoot! ser um aplicativo gratuito, acessível e considerado de fácil utilização, conforme já apresentado neste estudo, podese justificar que a experimentação não teve grande importância para os professores.

A "imagem" é outra característica de baixa significância. Essa variável representa o grau pelo qual a utilização de uma inovação é percebida como fator para melhorar a imagem ou o status de um indivíduo em um sistema social (MOORE; BENBASAT, 1991). Esse resultado corrobora a pesquisa realizada por Pérez et al. (2012), que também obtiveram esse resultado na investigação da inovação Moodle. Nessa mesma linha, a "visibilidade" não obteve significância neste estudo. Essa característica considera o grau pelo qual uma inovação se torna visível para outros indivíduos ou grupos dentro de uma organização (MOORE; BENBASAT, 1991). Esse resultado pode ser justificado pelo fato de que a adoção do Kahoot! não foi realizada pelos professores de administração ou contabilidade com o intuito de melhorarem sua imagem ou seu status no tocante aos demais professores do curso ou de outros cursos, assim como não são afetados pela visibilidade.

O "uso voluntário", que, neste estudo, considera o grau de percepção de uso voluntário ou espontâneo de uma inovação (MOORE; BENBASAT, 1991), não alcançou valor para ser significante. Possivelmente, se o uso do Kahoot! fosse estimulado pelos coordenadores das IES, essa alternativa seria significante. Gunter e Braga (2018) apontam, ainda, que a adoção da inovação para o ensino em sala de aula pode ser impactada pelas próprias restrições destacadas por professores quanto ao uso de tecnologias móveis nas instituições nas quais trabalham, o que também pode ter influência na baixa significância dessa característica. 
Por fim, analisa-se a "demonstração de resultado", que corresponde ao grau a partir do qual os resultados do uso de uma inovação são vistos como tangíveis (MOORE; BENBASAT, 1991). Conquanto essa variável não tenha sido significante a ponto de ser aceita, foi a que ficou mais próxima ao valor máximo (sig. de 0,08, e o máximo seria 0,05). Pode-se justificar que os professores não estão mensurando se, de fato, o desempenho dos alunos está evoluindo com sua adoção. Da mesma forma, embora existam pesquisas que apontem para o aumento de motivação e engajamento de alunos, há poucas pesquisas que, realmente, comprovam alguma evolução no desempenho final dos alunos.

\section{Considerações finais}

A partir da elaboração deste artigo foi possível responder à problemática de pesquisa proposta: Quais são as características percebidas pelos professores usuários do Kahoot! que contribuem para a sua adoção como ferramenta de apoio ao ensino em administração e contabilidade? A resposta para essa questão, no que concerne à característica percebida pelos professores de IES de Santa Catarina de administração e contabilidade para a adoção do Kahoot!, foi exclusivamente a "facilidade de uso", apresentando significância e impacto positivo, ou seja, quanto mais alto o nível de "facilidade de uso", maior o nível de adoção dessa inovação tecnológica.

Este estudo contribui para a discussão do uso de tecnologias no ensino de administração e contabilidade, sobretudo, no que se refere aos aplicativos e às tecnologias móveis, pois são pouco explorados na área (APOSTOLOU et al., 2016); à gamificação através do uso do Kahoot!, que apresenta lacunas para estudos empíricos (WANG; TAHIR, 2020); e, ainda, à difusão dessa inovação tecnológica, em um estudo brasileiro, que possui poucas pesquisas na área da inovação. O nível de explicação alcançado está de acordo com a teoria, porém sugere a participação de outros fatores no processo de adoção, o que também é apontado por Rogers (1983).

Como limitações deste estudo, destaca-se o número de participantes da pesquisa. Embora se tenha abordado todas as IES cadastradas no sistema ACAFE, apenas 30 professores responderam ao questionário. Porém destaca-se que as análises de regressão obtiveram significância mesmo com tal limitação. Sugere-se, portanto, que, em pesquisas futuras, sejam inclusos mais estados brasileiros ou outros países com o intuito de aumentar a amostra e, dessa forma, propiciar mais suporte empírico e mais robustez para as conclusões deste estudo.

Por fim, conclui-se que, para o aumento da motivação, participação e atenção dos alunos, faz-se necessário que os coordenadores das IES promovam um ambiente adequado para a utilização de tecnologias, e que as inovações para a educação apresentem facilidade de uso para que, assim, professores demonstrem tendência a utilizá-las e, consequentemente, haja aumento no engajamento dos alunos, com isso, auxiliando a superar as dificuldades encontradas na "crise da educação atual".

\section{Referências}


AGARWAL, Ritu; PRASAD, Jayesh. A field study of the adoption of software process innovations by information systems professionals. IEEE Transactions on Engineering Management, v. 47, n. 3, p. 295-308, 2000.

ANTUNES, Jeferson; DO NASCIMENTO, Verônica Salgueiro; DE QUEIROZ, Zuleide Fernandes. Metodologias ativas na educação: problemas, projetos e cooperação na realidade educativa. Informática na educação: teoria \& prática, v. 22, n. 1, 2019

APOSTOLOU, Barbara; DORMINEY, Jack W.; HASSELL, John M.; REBELE, James E. Accounting education literature review (2015). Journal of Accounting Education v. 35, p. 20-55, 2016. DOI: 10.1016/j.jaccedu.2016.03.002.

ARETIO, Lorenzo García. Necesidad de una educación digital en un mundo digital. RIED. Revista Iberoamericana de Educación a Distancia, v. 22, n. 2, 2019.

BORNAL, Eloisa Maistro et al. Contábil Quiz: Satisfação dos Estudantes de Ciências Contábeis Com o Uso de App no Processo de Ensino-Aprendizagem. Pensar Contábil, v. 21, n. 74, 2019.

CABERO ALMENARA, Julio; FERNÁNDEZ ROBLES, Bárbara. Las tecnologías digitales emergentes entran en la Universidad: RA y RV. RIED. Revista Iberoamericana de Educación a Distancia, 21 (2), 119-138., 2018.

DA CUNHA, Paulo Roberto et al. Oportunidades de melhoria na disciplina de Contabilidade Introdutória com a utilização do modelo Kano e da matriz de importância versus desempenho. Contextus-Revista Contemporânea de Economia e Gestão, v. 11, n. 2, 2013.

DABBAGH, Nada; FAKE, Helen; ZHANG, Zhicheng. Student Perspectives of Technology use for Learning in Higher Education. RIED. Revista Iberoamericana de Educación a Distância, v. 22, n. 1, p. 127-152, 2019.

DAVIS, Fred D.; BAGOZZI, Richard P.; WARSHAW, Paul R. User acceptance of computer technology: a comparison of two theoretical models. Management science, v. 35, n. 8, p. 982-1003, 1989.

DAVIS, Frederick Barton. Educational measurements and their interpretation. Wadsworth Publishing Company, 1964.

DE OLIVEIRA NETO, José Dutra; DE SOUSA GOMES, Gilvania; TITTON, Luiz Antônio. Using technology driven flipped class to promote active learning in accounting. Revista Universo Contábil, v. 13, n. 1, p. 49-64, 2017.

DE VARGAS, Sandra Belloli; SCHERER, Adriana Paula Zamin; GARCIA, Letícia Silva. As metodologias ativas no ensino da contabilidade: relato de experiências na sala de aula/The active methodologies in accounting education: report of experiences in the classroom. Brazilian Journal of Development, v. 6, n. 1, p. 3885 3905, 2020.

DELLOS, Ryan. Kahoot! A digital game resource for learning. International Journal of Instructional Technology and Distance Learning, v. 12, n. 4, p. 49-52, 2015.

FÁVERO, Luiz Paulo Lopes et al. Análise de dados: modelagem multivariada para tomada de decisões. 2009.

FEDERAÇÃO DAS INDÚSTRIAS DO ESTADO DE SANTA CATARINA - FIESC. Atividade econômica de SC cresce 2,54\% em 2019. Disponível em: < https://fiesc.com.br/pt-br/imprensa/atividade-economica-de-sc-cresce254-em-2019>. Acesso em: 29/02/2019.

FIELD, Andy. Descobrindo a estatística usando o SPSS-2. Bookman Editora, 2009.

GÓMEZ, Jennifer L.; MONROY, Liliana D. Gamification In Accounting Distance Education. Economy \& Business Journal, v. 12, n. 1, p. 208-215, 2018.

GUARDIA, Juan José et al. Innovation in the teaching-learning process: the case of Kahoot! On the Horizon, 2019.

GUNTER, Glenda A.; BRAGA, Junia de Carvalho Fidelis. Connecting, swiping, and integrating mobile apps affordances and innovation adoption in teacher education and practice. Educação em Revista, v. 34, 2018.

HAIR, Joseph et al. Fundamentos de métodos de pesquisa em administração. Bookman Companhia Ed, 2005. 
HAMARI, J.; KOIVISTO, J.; Y SARSA, H. Does Gamification Work? A Literature Review of Empirical Studies on Gamification (3025-3034). In: 47th Hawaii International Conference on System Sciences, IEEE. Hawaii, USA. 2014.

HIGGINS, Christopher A.; COMPEAU, Deborah R.; MEISTER, Darren B. From prediction to explanation: reconceptualizing and extending the perceived characteristics of innovating. Journal of the Association for Information Systems, v. 8, n. 8, p. 26, 2007.

HOERUP, Sharon Lynn. Diffusion of an innovation: Computer technology integration and the role of collaboration. 2001. Tese de Doutorado. Virginia Tech.

IGBARIA, Magid; GUIMARAES, Tor; DAVIS, Gordon B. Testing the determinants of microcomputer usage via a structural equation model. Journal of management information systems, v. 11, n. 4, p. 87-114, 1995

KEBRITCHI, Mansureh. Factors affecting teachers' adoption of educational computer games: A case study. British Journal of Educational Technology, v. 41, n. 2, p. 256-270, 2010.

LEAL, E. Fatores determinantes do uso de inovação tecnológica na educação à distância: um estudo com docentes dos cursos na área de negócios. 2012, 141 f. 2012. Tese de Doutorado. Tese (Doutorado em Administração) -Escola de Administração de Empresas, Fundação Getúlio Vargas, São Paulo. São Paulo.

LEWIS, William; AGARWAL, Ritu; SAMBAMURTHY, Vallabh. Sources of influence on beliefs about information technology use: An empirical study of knowledge workers. MIS quarterly, p. 657-678, 2003.

LOPES, Ana Paula et al. Gamification in education and active methodologies at higher education. 2019. In: Proceedings of EDULEARN19 Conference, $11^{\text {th }}, 2019$, Palma - Spain. Anais [...] Palma: IATED, 2019. DOI: 10.21125/edulearn.2019.0480

MALONE, Thomas W. Toward a theory of intrinsically motivating instruction. Cognitive science, v. 5 , n. 4 , p. 333-369, 1981.

MAROCO, João. Análise Estatística com o SPSS Statistics.: 7a edição. Report Number, Lda, 2018.

MAROCO, João; GARCIA-MARQUES, Teresa. Qual a fiabilidade do alfa de Cronbach? Questões antigas e soluções modernas?. Laboratório de psicologia, p. 65-90, 2006.

MOORE, Gary C.; BENBASAT, Izak. Development of an instrument to measure the perceptions of adopting an information technology innovation. Information systems research, v. 2, n. 3, p. 192-222, 1991.

NASU, Vitor Hideo; AFONSO, Luís Eduardo. Professor, Posso Usar o Celular? Um Estudo sobre a Utilização do Sistema de Resposta do Estudante (SRE) no Processo Educativo de Alunos de Ciências Contábeis. Revista de Educação e Pesquisa em Contabilidade (REPeC), v. 12, n. 2, 2018.

NASU, Vitor Hideo; NOGUEIRA, Daniel Ramos. Celulares a postos? Estudo sobre a percepção de alunos de ciências contábeis acerca do sistema de resposta de audiência (SRA). Enfoque: Reflexão Contábil, v. 39, n. 1, p. 01-19, 2020

NUNNALLY, Jum C. Psychometric Theory: 2d Ed. McGraw-Hill, 1978.

PEREZ, Gilberto et al. Tecnologia de informação para apoio ao ensino superior: o uso da ferramenta Moodle por professores de ciências contábeis. Revista de Contabilidade e Organizações, v. 6, n. 16, p. 143-164, 2012.

ROGERS, Everett M. Diffusion of innovation. 3 ed. New York: The Free Press, 1983.

SAHIN, Ismail. Detailed review of Rogers' diffusion of innovations theory and educational technology-related studies based on Rogers' theory. Turkish Online Journal of Educational Technology-TOJET, v. 5, n. 2, p. $14-23,2006$.

SILVA, Rui Jorge Rodrigues da; RODRIGUES, Ricardo Gouveia; LEAL, Carmem Teresa Pereira. Gamification in Management Education: A Systematic Literature Review. BAR-Brazilian Administration Review, v. 16, n. 2, 2019.

SWEETSER, Penelope; WYETH, Peta. GameFlow: a model for evaluating player enjoyment in games. Computers in Entertainment (CIE), v. 3, n. 3, p. 3-3, 2005.

TOLOMEI, Bianca Vargas. A gamificação como estratégia de engajamento e motivação na educação. EAD em foco, v. 7, n. 2, 2017. 
VALENTE, José Armando. Blended learning e as mudanças no ensino superior: a proposta da sala de aula invertida. Educar em Revista, n. 4, p. 79-97, 2014.

VENKATESH, Viswanath et al. User acceptance of information technology: Toward a unified view. MIS quarterly, p. 425-478, 2003.

VENKATESH, Viswanath; DAVIS, Fred D. A theoretical extension of the technology acceptance model: Four longitudinal field studies. Management science, v. 46, n. 2, p. 186-204, 2000.

VICK, I. (2019). Training professionals from three countries share their Kahoot!'ing experience (Vol. 2019). https://kahoot.com/blog/2019/09/10/top-training-tips-kahoot-around-world/: Kahoot!

WANG, Alf Inge; TAHIR, Rabail. The effect of using Kahoot! for learning-A literature review. Computers \& Education, v. 149, p. 103818, 2020.

Recebido em março de 2020.

Aprovado para publicação em junho de 2020

\section{Aline Espig}

Programa de Pós Graduação em Administração PPGAD, Departamento de Administração - Universidade Regional de Blumenau, FURB, Brasil, aline.espig@gmail.com

\section{Maria José Carvalho de Souza Domingues}

Programa de Pós Graduação em Administração PPGAD, Departamento de Administração - Universidade Regional de Blumenau, FURB, Brasil, mariadomingues@furb.br 